\title{
A Hypothesis of Symbiosis between Humans and Poliovirus as a Critical Evolutionary Step in Human Speciation
}

\author{
Adnan Saeed Amin* \\ Diplomate American Board of Psychiatry and Neurology, 5617 South Madelia Street \\ Spokane, WA 99223, USA
}

*Corresponding: aminadnansaeed2@gmail.com; telephone: 615-838-4161

\begin{abstract}
No single evolutionary event has been identified as the cause for the final emergence of our species. I propose that a mutation on CD155 receptor gene occurred to establish a symbiosis with poliovirus, which exerted its beneficial impact via RNA dependent non-genetic transgenerational inheritance, which caused a qualitative enhancement of cognitive functions. I posit that this mutation occurred in what we call, Anatomically Modern Humans, our immediate ancestor species and that the disruption of this symbiosis causes autism spectrum disorder.
\end{abstract}

Positive selection of CD155 to the extent of becoming a species defining characteristic, the chronology of autism spectrum disorder prevalence increase and continued increase, the multigenerational nature of RNA inheritance, the universal infection of humans by poliovirus and a very low associated mortality rate, and several other factors support this hypothesis.

Specific genetic, epidemiological and sperm miRNA content studies are suggested to test this hypothesis.

Keywords: symbiosis; speciation; humans; polio; CD155; PVR; evolution; ASD; autism

\section{Introduction}

Topics that may appear quite diverse at first glance constitute the background for the hypothesis presented. It is unknown what evolutionary event caused the final emergence of our species. It is well-established that symbiosis between species played a fundamental role in shaping evolution on this planet. Chloroplasts and mitochondria are prime examples ${ }^{1}$.

It is known that CD155 underwent rapid evolution in primates, exhibits only in humans a configuration capable of binding with the poliovirus with high affinity under natural circumstances, ${ }^{2}$ and this configuration was positively selected in humans to the extent of becoming a species defining characteristic ${ }^{3}$. Scientific literature is silent regarding the cause of this positive selection except the speculation that it was a result of binding to an unknown molecule but not to poliovirus ${ }^{3}$.

In the last 26 years the discovery of RNA dependent non-genetic transgenerational inheritance has created a tectonic shift in how biology is understood at fundamental levels. The idea that the brain activity through small RNAs, can impact the fate of several future generations went against a long-held, now inevitably abandoned, central tenet of biology ${ }^{4,5}$. It is now known that RNA dependent inheritance can transfer information regarding the experiences undergone by one individual organism to several future generations ${ }^{4-6}$, that this effect fades across a set number of generations ${ }^{7}$, that such inheritance can both originate from the CNS neurons as well as impact CNS neuronal development and cognitive functions in later generations ${ }^{5}$ - - four principles that have been demonstrated empirically in different species. Extracellular vesicles can transfer miRNA between tissues. Recent studies confirm that sperm RNA content analysis is possible. ${ }^{5,8}$

Poliovirus is an RNA virus and humans are it's only naturally occurring hosts 2 . Prior to the 19 th century no epidemics of acute flaccid paralysis due to poliomyelitis are recorded in any human culture ${ }^{10}$, the virus 
most likely infected nearly all humans universally ${ }^{9,10}$, had low rates of acute flaccid paralysis ${ }^{10}$ and an even lower rate of mortality, infected individuals at a young age ${ }^{9,10}$ and no poliomyelitis epidemics were recorded following European contact with previously separated populations including native American, native Australian, native Tasmanian and several others. Some authors report pre-contact poliomyelitis in Native American populations ${ }^{11}$. It has been opined that naturally acquired immunity to poliovirus existed at a very effective level in human population in the past ${ }^{12}$. The poliomyelitis epidemics which occurred from 1890s to 1950s in some countries were of an enigmatic nature compared with the past behavior of the virus ${ }^{9}$. It is now known that RNA viruses can alter and generate micro-RNAs in the host cells ${ }^{13}$. Genetic susceptibility to acute flaccid paralysis secondary to poliovirus infection in humans was never fully explored ${ }^{14}$.

Autism spectrum disorder is a neurodevelopmental disorder with a particular set of symptoms and deficits. In 2019, we have finally moved past the hypothesis that the increase in prevalence since 1980s in the US and in the rest of the world, was attributable entirely to better diagnosis ${ }^{15}$. It is now known that the prevalence rates are on the rise indeed, reaching about $4 \%$ of males aged 3-17 years, in the United States ${ }^{16-18}$. Developmental abnormalities begin to occur during fetal life in autism ${ }^{19}$. Autism is accompanied by miRNA dysregulation of primate specific miRNA ${ }^{20}$.

\section{Hypothesis}

I propose that the positive selection known to have been operating on the CD155, did so, in contrast to general belief, on the susceptibility to poliovirus infection, and not on the binding to another unknown molecule. A mutation in CD155 gene, also called PVR gene, generating a configuration that enabled this receptor to bind with the poliovirus with much higher affinity, occurred in the immediate ancestor species of current humans. This species was the one we have labeled as Anatomically Modern Humans (AMH). Described differently, what we call $\mathrm{AMH}$, is in fact made up of two separate species - the ancestral species with a CD155 capable of binding with the poliovirus with relatively low affinity in nature if at all, and an emergent species with a CD155 capable of doing so with a much higher affinity. This hypothesis postulates that this mutation and its subsequent positive selection were the last or one of the last, as well as one of the critical, events triggering the final emergence of our species.

As mentioned above, it is known that poliovirus infection can cause alterations in miRNA produced in human cells. What is unknown is whether such miRNA alterations are beneficial to the host or not. I posit that they are.

I further suggest that this symbiosis bestowed upon the host a qualitatively unprecedented benefit, as symbioses involving mitochondria and chloroplasts had exemplified in the distant evolutionary past. In this case however, the benefit was cognitive in nature. The mechanism of how this benefit was mediated involved the enhancement of the miRNA mediated non-genetic inheritance, with forward transmission from every individual that benefitted from this symbiosis to several future generations, the exact number of which is yet to be determined in our species. Something that can be visualized as a hypothetical deck of cards, stacked on top of each other, in an obliquely vertical manner, each card representing a separate generation of the host, covering part of several generations underneath but not all of them, every card in the middle and bottom of the deck being covered by several generations above it but not by all of them. The removal of one card from the middle of the deck, may or may not destabilize the structures sufficiently to disintegrate the stack. The higher the number of cards removed, higher the likelihood that the stack will disintegrate. Such disintegration can be deemed to be comparable to the disruption of critical cognitive functions (dependent upon miRNA based inheritance which is in turn dependent upon the continued symbiosis with poliovirus), culminating in the appearance of symptoms that have been labeled as autism.

\section{Evidence Supporting the Hypothesis}


The facts presented in the introductory paragraphs preceding the hypothesis section, now serve as supporting evidence, once the perspective from which they can be viewed has been informed by the above hypothesis. In summary, the data available regarding the evolution, positive selection and species specificity of CD155 clearly points to a critical beneficial impact of poliovirus on the host. The conclusions reached by others, based on the same data published so far, are prima facie incorrect in my opinion because they are based on the assumption that it is not possible for poliovirus to be of any benefit to humans $^{3}$.

Theoretical prediction of the nature and impact of a mutation causing human cognitive enhancement and speciation without any corresponding gross anatomical change, matching the mutation proposed in this article, was made decades ago. ${ }^{21}$

While keeping in mind the pitfalls associated with attempting to construct chronological correlations, the following question nevertheless demands an answer; did the rates of autism spectrum disorder start to exhibit a sudden and significant increase as the first ever human generation born to parents themselves protected against polio virus infection since infancy, began to enter the population mix? Can this hypothesis explain the continued increase in prevalence ever since?

When considered together the following observations also support the hypothesis. Humans are the only natural host, the virus caused a universal infection in human populations historically, did so in infancy, had a relatively low rate of paralysis and an even lower rate of mortality, and there is no notable record of poliomyelitis epidemics to have taken place in any human population prior to 1830 s, remarkably including the era following European contact with previously separated populations. In several countries of the world no massive poliomyelitis epidemics ever took place to our knowledge. These observations lend credence to the assertion that polio virus infection was pervasive and universal in all human populations including those that had been separated for about twenty to forty thousand years. This in turn supports the symbiosis hypothesis.

\section{Investigations to test the Hypothesis}

Following investigations are suggested to test the hypothesis. A determination of the timing of the last mutation on CD155 enabling it to act as high affinity receptor for poliovirus, is predicted to reveal that this event occurred in anatomically modern humans. Genome studies to be conducted on various specimens of anatomically modern human DNA, are predicted to reveal that anatomically modern humans are in fact two separate species. A species without, followed in time by a species with, the above-mentioned mutation, with corresponding behavioral differences among the two species. RNA content studies are predicted to show qualitative and quantitative differences in the sperm of those males exposed to poliovirus versus those never exposed to poliovirus versus those never exposed to poliovirus and born to parents themselves never exposed to poliovirus, confirming the slowly fading impact of the symbiosis across generations since its disruption. Also, based on this hypothesis, a significant increase in the prevalence of autism can be predicted in individuals with a set of parents and grandparents never exposed to poliovirus compared with even the highest observed rates so far in any country. Differences in prevalence of ASD between countries that disrupted poliovirus circulation earlier and completely versus those with a later onset of such efforts and only incomplete elimination of poliovirus circulation are also predicted. Other types of studies are also possible. 


\section{References}

1 Sagan L. On the origin of mitosing cells. J Theor Biol. 1967;14(3):225-IN6.

2 Ida-Hosonuma M, Sasaki Y, Toyoda H, Nomoto A, Gotoh O, Yonekawa H et al. Host range of poliovirus is restricted to simians because of a rapid sequence change of the poliovirus receptor gene during evolution. Arch Virol. 2003;148(1):29-44.

3 Suzuki Y. Ancient positive selection on CD155 as a possible cause for susceptibility to poliovirus infection in simians. Gene. 2006;373:16-22.

4 Posner R, Toker I, Antonova O, Star E, Anava S, Azmon E et al. Neuronal Small RNAs Control Behavior Transgenerationally. Cell. 2019;177(7):1814-1826.e15.

5 Benito E, Kerimoglu C, Ramachandran B, Pena-Centeno T, Jain G, Stilling R et al. RNA-Dependent Intergenerational Inheritance of Enhanced Synaptic Plasticity after Environmental Enrichment. Cell Reports. 2018;23(2):546-554.

6 Devanapally S, Ravikumar S, Jose A. Double-stranded RNA made in C. elegans neurons can enter the germline and cause transgenerational gene silencing. PNAS. 2015;112(7):2133-2138.

7 Houri-Zeevi L, Rechavi O. A Matter of Time: Small RNAs Regulate the Duration of Epigenetic Inheritance. Trends Genet. 2017;33(1):46-57.

8 Matsushima W, Brink K, Schroeder J, Miska E, Gapp K. Mature sperm small-RNA profile in the sparrow: implications for transgenerational effects of age on fitness. Environ Epigenet. 2019;5(2).

9 Nathanson N, Martin JR. The Epidemiology Of Poliomyelitis: Enigmas Surrounding Its Appearance, Epidemicity, And Disappearance1. Am J Epidemiol. 1979;110(6):672-92

10 Nathanson N, Kew OM. From Emergence to Eradication: The Epidemiology of Poliomyelitis Deconstructed. Am J Epidemiol. 2010;172(11):1213-29.

11 Martin DL. Health conditions before Columbus: paleopathology of native North Americans. West $J$ Med. 2002Jan;176(1):65-8 
12 Crawford CL. Naturally Acquired Immunity to Poliovirus: Historical Observations Have Been Ignored. J Infect Dis. 2006;194(11):1619

13 Rouha $\mathrm{H}$, Thurner $\mathrm{C}$, Mandl $\mathrm{CW}$. Functional microRNA generated from a cytoplasmic RNA virus. Nucleic Acids Res. 2010Dec;38(22):8328-37

14 Wyatt HV. Genetic Susceptibility: A Forgotten Aspect of Poliomyelitis. J Mol Genet Med. 2014;08(03)

15 Hertz-Picciotto I, Schmidt RJ, Krakowiak P. Understanding environmental contributions to autism: Causal concepts and the state of science. Autism Res. 2018;11(4):554-86.

16 Xu G, Strathearn L, Liu B, O'Brien M, Kopelman TG, Zhu J, et al. Prevalence and Treatment Patterns of Autism Spectrum Disorder in the United States, 2016. JAMA Pediatr. 2019Jan;173(2):153

17 Baio J, Wiggins L, Christensen DL, et al. Prevalence of Autism Spectrum Disorder Among Children Aged 8 Years - Autism and Developmental Disabilities Monitoring Network, 11 Sites, United States, 2014. MMWR Surveill Summ 2018;67(No. SS-6):1-23

18 Kogan MD, Vladutiu CJ, Schieve LA, Ghandour RM, Blumberg SJ, Zablotsky B, et al. The Prevalence of Parent-Reported Autism Spectrum Disorder Among US Children. Pediatrics. 2018;142(6)

19 Courchesne E, Pramparo T, Gazestani VH, Lombardo MV, Pierce K, Lewis NE. The ASD Living Biology: from cell proliferation to clinical phenotype. Mol Psychiatry. 2018;24(1):88-107.

20 Wu YE, Parikshak NN, Belgard TG, Geschwind DH. Genome-wide, integrative analysis implicates microRNA dysregulation in autism spectrum disorder. Nature Neurosci. 2016;19(11):1463-76.

21 Klein, R. G. Archeology and the evolution of human behavior. Evolutionary Anthropology: Issues, News, and Reviews, 2000:9(1), 17-36

I am the sole author of this manuscript.

Conflict of Interest Statement: I have nothing to disclose.

No funding source associated with the preparation of this manuscript.

Ethics committee approval not required given the nature of the manuscript. 